\title{
Die Entwicklung und der Mehrwert von Netzwerken anhand zweier Beispiele aus dem Projekt StiL - Studieren in Leipzig
}

Maria Förster, Priska Fronemann, Gretel Pfeiffer, Julia Protze, Mandi Strambowski, Marit Vissiennon

\section{Einleitung}

Durch den Qualitätspakt Lehre (QPL) sind im Verlauf der Förderzeit an der Universität Leipzig sowie an sächsischen Hochschulen verschiedene Netzwerke für unterschiedliche Zielgruppen entstanden, die einen wichtigen Beitrag für eine gelingende Projektentwicklung leisteten. Sie dienten nicht nur projektintern den Mitarbeiterinnen und Mitarbeitern zur Informationsgewinnung, zum Erfahrungsaustausch und zur gemeinschaftsbezogenen Identitätsbildung, sondern wirkten darüber hinaus auch in die Regelstrukturen der Hochschulen und hier speziell in die Universität Leipzig hinein. Dadurch wurde auch ein Kulturwandel in Bezug auf die Verbesserung der Qualität in der Lehre angestoßen.

Im folgenden Beitrag wird zunächst kurz auf die Rahmenbedingungen für und die Entwicklung von Vernetzung in Studieren in Leipzig (StiL) und seinen Teilprojekten eingegangen. Anschließend werden beispielhaft zwei Netzwerke vorgestellt, die im Rahmen des QPL an der Universität Leipzig entstanden sind: das Hochschul- und Mediendidaktische Netzwerk (HD-Netzwerk) und das StiL-Mentoring-Netzwerk.

Der Fokus liegt dabei auf folgenden Fragestellungen:

1. Wie ist das Netzwerk entstanden und welche Ziele wurden verfolgt?

2. Welche Entwicklungen waren im Netzwerk zu verzeichnen und welche Rahmenbedingungen haben dazu beigetragen?

3. Wie war das Netzwerk organisiert?

4. Welche Funktionen und welchen Mehrwert hatte das Netzwerk?

Im abschließenden Teil werden die Netzwerke hinsichtlich ihrer Struktur und Organisation verglichen und Schlussfolgerungen für die Bildung und Organisation zukünftiger Netzwerke gezogen. 


\section{Die Entwicklung der Vernetzung im Projekt StiL}

Die Maßnahmen in StiL waren im Projektantrag anhand dreier Säulen ausgerichtet, die sich aus dem Profil der Universität Leipzig ergaben und mit deren strategischen Entwicklungszielen korrespondierten: StiL zielte auf die Verbesserung der Infrastruktur und die Steigerung der Internationalität ab und sorgte für Reformimpulse durch innovative Projekte in Lehre und Studium. Entlang dieser drei Säulen (Infrastruktur, Internationalisierung, Innovation), aber auch säulenübergreifend, ergaben sich zahlreiche inhaltliche Bezüge zwischen den Teilprojekten.

In der ersten Förderphase des QPL (10/2011-09/2016) traten die Teilprojekte zunächst in einen Dialog und Austausch mit denjenigen Regelstrukturen, mit denen sich inhaltliche Bezüge innerhalb der Maßnahmen ergaben und/oder deren Zusammenarbeit eine erfolgreiche Zielerreichung beförderten oder sogar bedingten. Die Mitarbeitenden der Teilprojekte tauschten sich zunächst punktuell und individuell mit den betreffenden Stellen aus. Im Falle des Teilprojekts Mentoring betraf das auch die Vernetzung und die Zusammenarbeit untereinander, da die Mentorinnen und Mentoren über die Fakultäten der Universität Leipzig verteilt angebunden waren. Darüber hinaus fand Vernetzung mit weiteren QPL-Projekten in Sachsen und deutschlandweit statt, hier speziell durch die Teilnahme von Mitarbeitenden an Tagungen ${ }^{1}$. Ebenfalls war von Beginn an zu beobachten, dass StiL-Mitarbeitende sich aktiv an bestehenden festen Netzwerken beteiligten ${ }^{2}$ oder an der Initiierung oder Verfestigung von Netzwerken, die über das Projekt hinaus gingen, mitwirkten ${ }^{3}$.

1 Der deutschlandweite Austausch der QPL-Projekte wurde zum einen vom Bundesministerium für Bildung und Forschung (BMBF) durch Programmkonferenzen und themenspezifische QPL-Workshops unterstützt (vgl. BMBF 2020). Zum anderen veranstalteten QPL-Projekte deutschlandweit Fachtagungen, die (ab der zweiten Förderphase unterstützt durch Mittel für Austauschreisen) verstärkt von den Teilprojektmitarbeitenden besucht wurden.

2 Ein Beispiel dafür ist die regelmäßige Teilnahme von Mitarbeitenden des Mentorings, des Projektmanagements und des Career Service for Internationals am Beratungsnetzwerk des Projekts Plan B(eruf) (vgl. KOWA 2020).

3 Beispiele hierfür sind der Facharbeitskreis studentisches Peer-Learning (vgl. StiL 2020a), an dem das Tutoring-Kolleg beteiligt war (vgl. Schlimme et alt. in diesem Band), das sächsische Netzwerk zur qualitativen Evaluationsmethode Teaching Analysis Poll (TAP), die entsprechende bundesweite Arbeitsgruppe (AG) der Deutschen Gesellschaft für Hochschuldidaktik (DGHD) (vgl. DGHD AG 2020), die Initiierung und Mitarbeit des Teilprojekts Internationalisierung zu Hause und Mobilitätsförderung / Welcome Centre am Netzwerk Willkommen in Leipzig (vgl. Fabricius / Fromme in diesem Band) sowie die hochschulinterne, ressortübergreifende AG Service Learning (konzeptionelle und beratende Tätigkeit für die betroffenen Prorektorate [PBI und PET]) und der dazugehörige hochschulübergreifende Facharbeitskreis. 
Obwohl diese Entwicklung also schon in der ersten Förderphase einsetzte, gewann die Vernetzung von StiL-Mitarbeitenden untereinander, aber auch mit Mitarbeitenden anderer Projekte an der Universität Leipzig und hochschulübergreifend sowie mit den Regelstrukturen der Universität Leipzig im Übergang in die zweite Förderphase sowie in deren Verlauf (10/2016 - 12/2020) an Bedeutung. Die punktuelle Vernetzung mit einzelnen Bereichen wurde teilweise zu festen Netzwerken mit konstanter Mitgliederstruktur ausgebaut. Der Austausch erfolgte regelmäßig und die jeweiligen Maßnahmen wurden zunehmend den Zielgruppen der weiteren Netzwerkmitglieder zugänglich gemacht. Zum Teil wurden gemeinsam Angebote entwickelt und/oder durchgeführt.

Das Projektmanagement bildete dabei die Schnittstelle zwischen den Teilprojekten und der Projektleitung sowie weiteren zentralen und dezentralen Akteuren an der Universität Leipzig. Es war zudem Ansprechpartner für den Projektträger, das Deutsche Zentrum für Luft- und Raumfahrt (DLR). Zu den Aufgaben des Projektmanagements zählten neben Monitoring, Qualitätssicherung und Steuerung des Projekts die Begleitung und Beratung der Teilprojekte, die Konzeption von Maßnahmen sowie Förderung von Kommunikation und Vernetzung. Das Projektmanagement unterstützte die Teilprojekte dabei, gemeinsame Bezüge sichtbar zu machen und zu stärken, und regte weitere zielgerichtete Vernetzung zwischen Teilprojekten und in die Universität Leipzig hinein an. Dazu wurden unter anderem Auftakttreffen zu Beginn der Förderphasen mit allen StiL-Beteiligten organisiert und regelmäßig Projektgespräche mit den Teilprojekten durchgeführt. ${ }^{4}$ Das Projektmanagement vertrat StiL zudem im sächsischen QPL-Netzwerk (sQPLnet). Dieses entstand 2013 und verband die 13 sächsischen QPL-Projekte. ${ }^{5}$ Das Netzwerk wurde vor allem als Pool für Experten und Expertinnen zu verschiedenen Bereichen (zum Beispiel Beratung oder Qualitätsentwicklung) genutzt, aber auch für den Transfer von Good Practice sachsenweit. ${ }^{6}$

4 Weitere Instrumente waren ein Projekthandbuch und der StiL-Moodle-Kurs. Das Projekthandbuch beinhaltete Informationen zu den Teilprojekten sowie wichtigen organisatorischen Prozessen und wurde den Teilprojekten regelmäßig in aktualisierter Ausgabe zur Verfügung gestellt. Der StiL-Moodle-Kurs war ein Online-Kurs auf Moodle, der zentralen Lernplattform der Universität Leipzig (vgl. Moodle 2020). Es wurden Dokumente zur Verfügung gestellt, über Foren kommuniziert sowie in Wikis und Datenbanken kollaborativ Inhalte erarbeitet.

5 Neben Einzelvorhaben waren mehrere sächsische Hochschulen am Verbundprojekt Lehrpraxis im Transfer plus (LiT+) beteiligt (vgl. QPL 2020).

6 Ein konkretes Ergebnis der Zusammenarbeit des Netzwerks war 2016 die Tutoring-Leitlinie, die zu Beginn der zweiten Förderphase an der Universität Leipzig eingeführt wurde. Sie beinhaltete Hinweise zur Einstellung und Betreuung von Tutorinnen und Tutoren, die für die Umsetzung von StiL-Tutorien an der Universität Leipzig galten (vgl. StiL 2020b). 
In der zweiten Förderphase führte das Projektmanagement mit den Koordinierungsgruppen ${ }^{7}$ ein neues regelmäßiges Austauschformat ein, das zweimal pro Kalenderjahr stattfand und von den StiL-Mitarbeitenden rege genutzt wurde. Ein StiL-Lenkungskreis wurde eingerichtet, der die Projektentwicklung beratend und begleitend unterstützte ${ }^{8}$. Weiterhin wurden Projektergebnisse durch Austausch mit Mitarbeitenden anderer Projekte, bei Tagungsteilnahmen und eigenen Beiträgen bei Tagungen deutschlandweit vermittelt.

\section{Beispiele für Netzwerke in StiL}

In StiL entwickelten sich Netzwerke, die über einen längeren Zeitraum aktiv waren sowie auf verschiedenen Ebenen wirkten. Zwei dieser Netzwerke sollen hier beispielhaft vorgestellt werden: Das Mentoring-Netzwerk verband die Mitarbeitenden des Teilprojekts Mentoring, die an 12 der 14 Fakultäten der Universität Leipzig angebunden waren. Im Hochschul- und Mediendidaktischen Netzwerk (HD-Netzwerk) waren mit der LaborUniversität und dem Tutoring-Kolleg zwei StiL-Teilprojekte sowie das Projektmanagement vertreten.

\section{Das Mentoring-Netzwerk als Community of Practice ${ }^{9}$}

An 12 der 14 Fakultäten der Universität Leipzig wurden über das Projekt StiL Mentoring-Stellen geschaffen. Die wissenschaftlichen Mitarbeitenden wurden an den Fakultäten damit beauftragt, eine Beratungsstruktur zu schaffen, die eine Lücke zwischen zentralen Angeboten der Studienberatung und institutsspezifischen

7 Koordinierungsgruppen waren Austauschformate auf Arbeitsebene. Sie dienten der gezielten Abstimmung von Querschnittsthemen und konkreten Maßnahmen sowie dem Transfer von Ergebnissen und Konzepten. Neben StiL-Mitarbeitenden wurden je nach inhaltlicher Ausrichtung Experten und Expertinnen aus den Regelstrukturen der Universität Leipzig oder anderen Projekten an der Universität Leipzig eingeladen. Die Koordinierungsgruppen gaben Impulse, die die Projektmitarbeitenden anschließend in ihre Arbeit übertrugen. Als Ergebnis formierten sich zum Teil Arbeitsgruppen, die aufgrund ihrer Tätigkeiten, Zielgruppen und/oder institutionellen Anbindung verstärkt miteinander kooperierten und sich zu Communities of Practice (siehe unten) entwickelten.

8 Der Lenkungskreis war ein mit Vertreterinnen und Vertretern aus Senatsmitgliedern, Lehrenden, Studierendenschaft und Zentralverwaltung sowie der Stabsstelle Qualitätsentwicklung in Lehre und Studium und der oder dem Gleichstellungsbeauftragten besetztes Gremium.

9 Die drei wesentlichen Merkmale einer Community of Practice sind gemeinsames Wissensgebiet, Zusammengehörigkeitsgefühl und praktische Erfahrung (vgl. Lave / Wenger 1991). 
Angeboten schließen sollte. Darüber hinaus waren die Mentorinnen und Mentoren in den Themenfeldern Studienorientierung, Beratung und Studienbegleitung, Internationalisierung, Berufsorientierung, Qualitätssicherung und Evaluation tätig. Durch die Übernahme dieser Querschnittsthemen etablierten die wissenschaftlichen Mitarbeitenden eine neue Serviceeinrichtung in den Fakultäten.

Wie ist das Netzwerk entstanden und welche Ziele wurden verfolgt?

Noch vor dem offiziellen Auftakttreffen des Projekts StiL fand ein erstes von den Mentorinnen und Mentoren initiiertes Treffen der im Teilprojekt neu eingestellten Mitarbeitenden statt. Da die Gruppe durch die Rollenbezeichnung ${ }^{10}$, die Projektaufgaben sowie die gleichzeitige Anbindung an die Fakultäten und das Projekt StiL viele gemeinsame Anknüpfungspunkte hatte, lag diese Form der Vernetzung nah. Ziel der turnusmäßig stattfindenden Mentoring-Netzwerktreffen war es besonders zu Projektbeginn, sich in die neue Aufgabe einzufinden, die im Antrag vorgegebenen Ziele in konkrete Maßnahmen zu übersetzen sowie gemeinsame Schnittpunkte zu identifizieren. Diese Ausrichtung war insofern für die Beteiligten entscheidend, da die Richtung, in welche die Mentoring-Tätigkeit gehen sollte und konnte, noch ausgelotet werden musste. Neben der Entwicklung gemeinsamer fakultätsübergreifender Angebote bestand ein weiteres Ziel der Netzwerktreffen im interfakultären Informations- und Erfahrungsaustausch und somit auch in der Qualifizierung und Professionalisierung der Mentorinnen und Mentoren.

Welche Entwicklungen waren im Netzwerk zu verzeichnen und welche Rahmenbedingungen haben dazu beigetragen?

Das Mentoring-Netzwerk durchlief verschiedene Phasen, die von der Vernetzung, Konzeptionierung und Pilotierung bis zur Konsolidierung reichten.

a. Vernetzungsphase: Die anfangs regelmäßig aller zwei bis drei Monate stattfindenden Treffen boten den Mentorinnen und Mentoren die Gelegenheit, sich untereinander kennen zu lernen und wurden auch bald zu einer Plattform für andere universitäre Einrichtungen und Projekte, sich und ihre Arbeit vorzustellen. Diese Phase war maßgeblich davon geprägt, Ansprechpersonen und Kooperationspartner und -partnerinnen kennenzulernen, sich über Erfah-

10 Die Bezeichnung Mentoring ist hier etwas irreführend, da das Projekt kein Mentoring im klassischen Verständnis einer 1:1-Betreuung darstellt, sondern eher dem Konzept einer Studienverlaufsberatung ähnelt. 
rungen auszutauschen sowie Möglichkeiten der Zusammenarbeit auszuloten und zu erproben. ${ }^{11}$

b. Konzeptionierungs- und Erprobungsphase: Nach der Identifizierung von gemeinsamen Schnittpunkten widmeten sich die Mentorinnen und Mentoren in einer zweiten Phase der konkreten Konzeptionierung sowie Pilotierung erster überfakultärer Projekte, die aus kleineren Arbeitsgruppen heraus innerhalb des Netzwerks entstanden waren. So entwickelten sich zum Beispiel die beiden Peer-Mentoring-Programme BeBuddy! für internationale Studierende und das Lehramtsmentoring. ${ }^{12}$ Neben dem regelmäßigen Wissens- und Erfahrungsaustausch fokussierten sich die Mentorinnen und Mentoren in dieser Phase stark auf die Entwicklung sowohl fakultärer als auch gemeinsamer Angebote. Hierbei wurde während der ersten Förderphase verstärkt das im Projektantrag beschriebene Ziel, Lücken im Beratungsangebot der Universität Leipzig zu schließen, verfolgt. Aspekte wie strategisches Vorgehen, Nachhaltigkeit oder die Verknüpfung mit bereits bestehenden Angeboten rückten erst später in den Fokus der Arbeit. Vortragsreihen, Workshops, Informationsveranstaltungen sowie Peer-Programme wurden konzipiert, pilotiert und evaluiert sowie je nach Ergebnis weitergeführt oder eingestellt.

c. Konsolidierungsphase: Die zweite Förderphase war zunächst von der Fortführung bestehender fakultätsinterner und fakultätsübergreifender Angebote geprägt. Das Netzwerk arbeitete in seiner nun etablierten Struktur weiter und die Ergebnisse daraus fanden Anwendung in den fakultären Strukturen. Gemeinsame thematische Schwerpunkte wurden in den Arbeitsgruppen vertieft. Die Häufigkeit der Netzwerktreffen wurde den Bedarfen der Mentorinnen und Mentoren angepasst und im Laufe der Zeit reduziert. Die Arbeit gewann insgesamt an Routinecharakter und richtete sich wesentlich an den Aufgaben innerhalb der Fakultäten aus. Die Netzwerktreffen wurden vorwiegend für den Informationsaustausch sowie anliegende Handlungsabstimmungen genutzt.

d. Phase einer gemeinsamen strategischen Ausrichtung: Mit dem nahenden Ende der zweiten Förderphase bekam das Mentoring-Netzwerk einen neuen Anschub. Gemeinsame strategische Überlegungen zur Fortführung der mitt-

11 Beispielsweise fanden in Kooperation mit dem Career Service der Universität Leipzig Veranstaltungen im Bereich der Berufsfeldorientierung für einzelne Fächergruppen (unter anderem Berufsorientierung für Biologinnen und Biologen und Öffentlichkeitsarbeit für Sprachwissenschaftlerinnen und -wissenschaftler) statt.

12 Für weitere Informationen zu den studentischen Peer-Mentoring-Programmen siehe Dimmer et al. in diesem Band. Ein weiteres Beispiel für ein fakultätsübergreifendes Projekt ist die Naturwissenschaftliche Forschungswerkstatt (vgl. Holzheu et al. in diesem Band). 
lerweile etablierten Tätigkeiten der Mentorinnen und Mentoren regten einen Prozess an, in dem sich die Gruppe noch einmal auf neue Art formieren und reflektieren konnte. Im Fokus standen dabei die zukünftige Ausrichtung des Mentorings sowie die Ziele und das Selbstverständnis des Netzwerks. Dazu war ein intensiver Diskussions- und Aushandlungsprozess in der Gruppe nötig, der maßgeblich durch intern neu eingeführte Formate wie Klausurtagungen und Visionstage unterstützt wurde. In diesem Prozess konnte die Gruppe ihren Mehrwert in struktureller und gesamtuniversitärer Hinsicht verorten. Die Überlegungen mündeten in einem Mentoring-Zukunftskonzept. In den vorhergehenden Phasen kooperierten die Mentorinnen und Mentoren zwar bereits intensiv, agierten jedoch immer aus ihren fakultären Bedarfen und Zielen heraus. Nun stand die Gruppe vor der Herausforderung, sich als Gesamtgruppe zu definieren und ein Commitment herzustellen. Damit einher ging eine Profilschärfung des Mentorings sowie eine Stärkung des Zusammengehörigkeitsgefühls.

Wie war das Netzwerk organisiert?

Die Organisation des Mentoring-Netzwerks zeichnete sich insbesondere durch zwei Aspekte aus: geteilte Verantwortlichkeiten und Freiwilligkeit. Die Netzwerktreffen wurden jeweils von zwei Mentorinnen oder Mentoren unterschiedlicher Fakultäten ausgerichtet. Sie übernahmen sowohl die Organisation der Treffen sowie die Moderation der im Vorfeld abgefragten und von ihnen selbst vorgeschlagenen Tagesordnungspunkten und die Ergebnissicherung durch Protokollierung. Inhaltlich ging es vor allem um aktuelle fakultätsübergreifende Themen und Informationen, neue Projekte der Mentorinnen und Mentoren sowie anderer Einrichtungen wurden vorgestellt, Projekterfahrungen geteilt sowie Methoden, bewährte Vorgehensweisen und Problemlösungen besprochen. Zudem war es bei gemeinsamen Themen notwendig, Aufgaben zu verteilen und nächste Schritte abzustimmen. ${ }^{13}$ Bei Themen, in denen ein Interesse der Gruppe bestand, sich umfangreicher auszutauschen, wurden ab 2018 sogenannte Praxiswerkstätten eingeführt. Dieses Format gab Raum, um eigene Erfahrungen in der Gruppe zu reflektieren, sich neue Anregungen bei anderen Mentorinnen und Mentoren zu holen sowie konkrete Tools oder Vorlagen auszutauschen. Kleinere Aufgaben, welche die gesamte Gruppe betrafen und sich aus dem Treffen ergaben, wurden im Anschluss in kleinere Arbeitsgruppen gegeben.

13 Zum Beispiel für die Planung der Öffentlichkeitstage der Universität Leipzig, der Willkommenswoche oder einzelner gemeinsamer Veranstaltungen. 
Auch ohne externe Vorgabe durch Vorgesetzte oder die Projektleitung war die Teilnahme an den Treffen stets hoch. Es etablierten sich schnell verschiedene Verfahren und Formen für die Abstimmung und die Kommunikation in der Gruppe. Zum Beispiel wurde die sich je nach Phase der Gruppe ändernde Häufigkeit der Treffen stets demokratisch abgestimmt. Auch wenn die Beteiligung an und während der Treffen zwischen sehr aktiv bis zurückhaltend variierte, wurde der Wissens- und Erfahrungsaustausch von den Teilnehmenden als bereichernd und als Möglichkeit zur Qualifizierung und Professionalisierung der eigenen Rolle wahrgenommen.

Eine Reflexion des Netzwerkes führt zu dem Schluss, dass

1. die Teilnahme und Beteiligung am Netzwerk in einem engen Zusammenhang mit der individuellen Relevanz der Themen für die eigene Arbeit an den Fakultäten stand,

2. die Intensität der Netzwerkarbeit sehr von gemeinsamen Anliegen, Themen und Zielen abhing und diese Gemeinsamkeiten in einer Gruppe nicht künstlich herstellbar waren ${ }^{14}$ und

3. die Netzwerkarbeit sehr stark von der Eigeninitiative und der aktiven Beteiligung der Teilnehmenden lebte. Dabei brauchte es Schlüsselpersonen, die das Netzwerk hegten, pflegten und bewässerten („Gardening“汭 .

\section{Welche Funktionen erfüllte das Netzwerk?}

Das Mentoring-Netzwerk erfüllte unterschiedliche Funktionen sowohl für seine Mitglieder als auch für den gesamtuniversitären Kontext. Besonders dann, wenn Arbeitsaufträge einzelner Mentorinnen und Mentoren an den Fakultäten ähnlich waren oder zentrale Aufgaben, beispielsweise durch die Projektleitung, an das Netzwerk herangetragen wurden, kam das Netzwerk als Ort des Wissens- und Erfahrungsaustauschs zum Tragen. Häufig gab es in den Netzwerktreffen Initialzündungen für verschiedene Projekte, die dann in die Fakultäten oder den gesamtuniversitären Kontext hineinwirkten. Die Expertise des Netzwerks wurde auch von anderen Netzwerken und Akteuren innerhalb und außerhalb der Universität Leipzig regelmäßig nachgefragt.

14 In der Konsolidierungsphase gab es dabei zeitweise nur wenige Treffen des Mentoring-Netzwerks im Jahr, da der Bedarf nicht sehr groß war. Die Mentorinnen und Mentoren waren mit der konkreten Durchführung von Maßnahmen und deren Organisation ausgelastet.

15 Communities of Practice müssen dem Konzept von Lave und Wenger (1991) nach „,begärtnert“ (,Gardening“) und ,genährt“ (,Nurturing“) werden. 


\section{Die LaborUniversität im universitätsweiten Hochschul- und Medien- didaktischen Netzwerk}

Die LaborUniversität war in der ersten Förderphase des QPL auf die Förderung und Begleitung von Lehrinnovationen (Lehr-Lernprojekte) konzentriert. Zudem erweiterten die Mitarbeiterinnen bereits damals den Radius hochschuldidaktischer Aktivitäten; auch im Sinne des Transfergedankens der Lehr-Lernprojekte. In der zweiten Förderphase ab Oktober 2016 wurden einhergehend mit einer breiteren personellen Ausstattung das Portfolio um die professionelle Begleitung und Beratung von Lehrenden zu hochschuldidaktischen Fragen entlang hochschuldidaktischer Schwerpunktthemen ${ }^{16}$ sowie die Unterstützung bei längerfristigen Prozessen wie der Studiengangentwicklung erweitert. Die LaborUniversität arbeitete dafür eng mit dem sachsenweit tätigen Verbundprojekt Lehrpraxis im Transfer ${ }^{\text {plus }}$ (LiT+) sowie dem Hochschuldidaktischen Zentrum Sachsen (HDS) zusammen. Aus dieser Zusammenarbeit entstand das Hochschul- und Mediendidaktische Netzwerk, auf das im Folgenden genauer eingegangen wird.

Wie ist das Netzwerk entstanden und welche Ziele wurden verfolgt?

An der Universität Leipzig waren seit Förderbeginn des QPL zwei hochschuldidaktische Projekte verortet: Das StiL-Teilprojekt LaborUniversität sowie das sächsische Verbundprojekt Lehrpraxis im Transfer (LiT) ${ }^{17}$. Von Beginn an war es das Interesse beider Projekte, sich auszutauschen und im gemeinsamen Feld der Hochschuldidaktik voneinander zu lernen. Beide Projekte legten einen Fokus auf die Förderung von Lehr-Lernprojekten. Die LaborUniversität profitierte dabei von LiT in Bezug auf dessen hochschulübergreifende Vernetzung und Erfahrungen, die hier mit verschiedenen Maßnahmen neben der Lehrprojektförderung gemacht wurden. LiT erhielt durch die enge Anbindung der LaborUniversität an die Universität Leipzig und die zahlreichen universitätsinternen Lehr-Lernprojekte einen tieferen Einblick in die Lehre an der Universität Leipzig. Schnell verständigten sich beide Projekte zusammen und mit der Projektleitung darauf, abgestimmt auf die Fakultäten und weitere zentrale Akteure der Hochschuldidaktik an der Universität Leipzig zuzugehen. Die ab 2013 gemeinsam durchgeführten Auftaktgespräche mit Fakultätsleitungen, weiteren identifi-

16 Diese waren: Kompetenzorientierung, Interdisziplinarität, Praxisbezug, Forschungsbezug, Einsatz digitaler Medien sowie Umgang mit Heterogenität in der Lehre.

17 Das Projekt Lehrpraxis im Transfer hieß ab Beginn der zweiten Förderphase Lehrpraxis im Transfer ${ }^{\text {plus }}$. 
zierten (hochschuldidaktischen) Mitwirkenden ${ }^{18}$ sowie zentralen Einrichtungen dienten einer ersten Bedarfserhebung zu hochschuldidaktischen Themen und Maßnahmen. Weitere Austauschgespräche mit den Akteurinnen und Akteuren zielten darauf ab, die zukünftige Zusammenarbeit in Bezug auf hochschuldidaktische Angebote zu entwickeln. Gemeinsam entstand die Idee, für Lehrende der Universität Leipzig eine Übersicht über die hochschuldidaktischen Angebote zu schaffen und einen gemeinsamen Internetauftritt einzurichten. ${ }^{19}$ In der Folge entstanden zunächst erste gemeinsame Informationsmaterialien. In diese Phase fielen die ersten Schritte des hochschuldidaktischen Netzwerks.

Das vom Personaldezernat im März 2015 initiierte Vernetzungstreffen der Anbieter von Fortbildungen war ein weiterer Schritt hin zu einem festen Netzwerk. Es hatte zum Ziel, sich über die fachliche Arbeit, Ziele, Zielgruppen und Umsetzung auszutauschen. In den darauffolgenden Treffen wurde erarbeitet, wie die Akteure koordiniert werden könnten, um den Zielgruppen möglichst einfach die jeweils passenden Angebote zugänglich zu machen. 2017 wurde dann erstmals ein Treffen des gesamtuniversitären Hochschul- und Mediendidaktischen Netzwerks ${ }^{20}$ unter Vorsitz des Prorektors für Bildung und Internationales organisiert, um die verschiedenen bestehenden Ansätze kontinuierlich abzustimmen und zu synchronisieren. Ziele des Netzwerks waren:

- ein gemeinsames Verständnis über Hochschuldidaktik zu entwickeln,

- Doppelstrukturen zu vermeiden,

- die Sichtbarkeit für die Zielgruppe zu erhöhen, um den Zugang für die Nutzerinnen und Nutzer zu erleichtern,

- den Stellenwert von Lehre an der Universität Leipzig zu erhöhen sowie

- die Lehre als solche zum Thema zu machen.

18 Dazu gehörten das Sachgebiet Wissenschaftliche Weiterbildung, das HDS, das Referat Personalentwicklung, die Tutorinnen-und-Tutoren-Qualifizierung, das StiL-Teilprojekt E-Teaching, der E-Learning-Service und die Research Academy Leipzig (ESF-Projekt Kompetenzschule ELSYS).

19 Ein gemeinsamer Internetauftritt war erst später (2018) mit der grundlegenden Überarbeitung der Universitätswebsite möglich. Neben den Bereichen Studium und Forschung wurde nun auch der Bereich Lehre eingerichtet.

20 An der Universität Leipzig betraf das auf zentraler Ebene Mitarbeitende des Sachgebietes der Wissenschaftlichen Weiterbildung, der Personalentwicklung, der Stabsstelle für Qualitätsentwicklung in Lehre und Studium, der Research Academy Leipzig und des E-Learning-Services (seit 2019 inklusive des Projekts Digitale Hochschulbildung in Sachsen) sowie auf Ebene der Fakultäten das Medizindidaktische Zentrum. Ebenso vertreten war das HDS, das Verbundprojekt LiT+ und seitens StiL die LaborUniversität, das Tutoring-Kolleg sowie das Projektmanagement. 
Welche Entwicklungen waren im Netzwerk zu verzeichnen und welche Rahmenbedingungen haben dazu beigetragen?

Die wichtigste Rahmenbedingung bildete zu Beginn neben der räumlichen Nähe ${ }^{21}$ vor allem der gemeinsame Wille, das Projekt an der Universität Leipzig aufzubauen und das Thema Lehre nachhaltig zu etablieren. Dadurch wurde auf informeller Ebene ein reger kollegialer Austausch und diverse Perspektivverschränkungen ermöglicht. Es entstand ein zunächst wöchentlicher Jour fixe, bei welchem Transparenz zwischen den Mitarbeitenden über aktuelle Maßnahmen und Projekte hergestellt sowie sich über inhaltliche und strukturelle Entwicklungen ausgetauscht wurde. In dieser frühen Phase des QPL, die von der Suche nach passenden Maßnahmen und Themen für die wirksame Erreichung des Kulturwandels in der Lehre geprägt war, wirkte dies sehr bereichernd. Das Thema Lehre und die Hochschuldidaktik als solche fanden an der Universität Leipzig bis dahin noch wenig Beachtung. Initial setzte die Planung und Umsetzung des ersten Tages der Lehre 2013 an diesen Zielen an. Abstimmungsprozesse und Kommunikationsroutinen wurden in der kooperativen Konzeption und Umsetzung dieser Veranstaltung etabliert.

Das Hochschul- und Mediendidaktische Netzwerk wurde in der zweiten Förderphase des QPL durch die Unterstützung der Hochschulleitung mit dem Auftakttreffen des Hochschuldidaktischen Netzwerks $2017^{22}$ formalisiert und um zusätzliche relevante Akteure erweitert. Wichtige Meilensteine waren anfänglich die Verständigung auf gemeinsame Ziele und Strategien, eine gute Abstimmung zu deren gegenseitiger Ergänzung und Verschränkung sowie die Konzipierung gemeinsamer Veranstaltungen wie den Tagen der Lehre ${ }^{23}$.

Bisher konnten verschiedene Vorhaben im Netzwerk realisiert werden. Dies betraf insbesondere die bessere Sichtbarkeit und Abstimmung untereinander zum Vermeiden von Doppelstrukturen und das Bilden von Synergien. Es wurde ein gemeinsamer Webauftritt Lehren an der Universität (2019) geschaffen, um

21 Die StiL-Teilprojekte LaborUniversität und E-Teaching, das Projektmanagement, das Verbundprojekt LiT+ sowie weitere Mitarbeitende des Prorektors für Bildung und Internationales teilten sich Büros auf einem Gang.

22 Die stärkere Verzahnung zwischen Hochschul- und Mediendidaktik war von Beginn an ein wichtiges Thema. Im Projekt StiL gab es in der ersten Förderphase des QPL das mediendidaktische Teilprojekt E-Teaching, welches in der zweiten Förderphase als Querschnittsthema in die LU integriert wurde.

23 Der Tag der Lehre wird seit 2013 an der Universität Leipzig veranstaltet, seit 2017 im jährlichen Turnus. Eingeladen sind alle Lehrenden, Studierenden und Mitarbeitenden der Universität und weiterer Leipziger Hochschulen zu einem gemeinsamen Austausch über fachspezifische und fächerübergreifende Fragen des Lehrens und Lernens. Der Tag der Lehre macht das vielfältige Engagement und innovative Ideen in der Lehre an der Universität Leipzig sichtbar (vgl. Universität Leipzig 2020). 
das Thema Lehre sichtbarer zu machen. Auf dieser Website wurde zudem ein gemeinsamer Veranstaltungskalender etabliert und der Zugang zur hochschulinternen Fortbildungsdatenbank verbessert ${ }^{24}$. Ein gemeinsamer interner Kalender führte zudem zu einem stärker abgestimmten Programmangebot. Des Weiteren wurden projektübergreifende Veranstaltungen und Angebote konzipiert und umgesetzt. Zu nennen sind hier neben dem Tag der Lehre die hochschuldidaktische WERKSTATT zur Lehrveranstaltungsplanung, das Modell und die darin verankerten Angebote zur hochschuldidaktischen Begleitung der Studiengangentwicklung, hochschuldidaktische Handreichungen sowie Tipps für die Lehre im gemeinsamen Webauftritt.

Die Corona-Pandemie und das damit verbundene digitale Sommersemester 2020 erforderte eine neuartige, weil vorwiegend digitale, Vernetzung der Akteure. Dabei wurde die Struktur des Netzwerks weiter gefestigt und besonders die Zusammenarbeit mit den Mitarbeitenden des E-Learning Service und des dort verorteten Verbundprojekts Digitale Hochschulbildung intensiviert. Der Webauftritt wurde für das digitale Sommersemester um didaktische und medientechnische Tipps sowie (medien-)didaktische Handreichungen für Lehrende erweitert. In dem neu eingerichteten Moodle-Kurs Lehre.digital konnten sich die Lehrenden untereinander bezüglich ihrer Fragen und Erfahrungen in einem Forum austauschen. Zudem wurden sie von den medien- und hochschuldidaktischen Mitarbeitenden im Kurs sowie durch individuelle Online-Beratungen unterstützt, zum Beispiel mittels digitaler Hospitationen und E-Coachings.

\section{Wie war das Netzwerk organisiert?}

Das Netzwerk entwickelte sich als Bottom-up-Prozess der an einer stärkeren Sichtbarkeit und Wirksamkeit interessierten QPL-Projekte. Zunächst tauschten sich die hochschul- und später zunehmend auch die mediendidaktischen Akteure an der Universität Leipzig aus. Ab der zweiten Förderphase des QPL und der offiziellen Einrichtung der Hochschul- und Mediendidaktischen Netzwerktreffen, traf sich das Netzwerk im drei- bis sechsmonatigen Zyklus unter der Leitung des Prorektors für Bildung und Internationales. Zwischen den physischen Treffen erfolgte der Austausch in einem gemeinsamen Kurs auf der Lernplattform Moodle, auf der die Protokolle der Treffen abgelegt sowie in Foren untereinander kommuniziert wurden. In der Zusammenarbeit entstanden verschiedene Arbeitsgruppen, die sich wiederum zeitweise in kleine Untergruppen für einzelne Aufgabenpakete

24 Die Angebote sind in der Fortbildungsdatenbank der Universität Leipzig unter Hochschulund Mediendidaktik an der Universität Leipzig gebündelt und können dort von Lehrenden gebucht werden (vgl. https://fortbildung.uni-leipzig.de). 
aufteilten. Dieses Prinzip erwies sich auch in der Zeit der Corona-Pandemie als wirksame Arbeitsweise. Regelmäßige Absprachen zur Erhöhung der Transparenz, das Engagement und die gemeinsame Vision der Beteiligten sowie die Steuerung durch den Prorektor waren dabei essenziell für das Gelingen des Netzwerks.

\section{Welche Funktionen erfüllte das Netzwerk?}

Neben den bereits beschriebenen positiven Effekten für die Etablierung und Sichtbarmachung der Hochschuldidaktik selbst, wirkte das Netzwerk in Richtung der gemeinsamen Vision und förderte und ermöglichte einen intensiven Austausch über Themen der Lehre an der Universität Leipzig insgesamt sowie an den Fakultäten, in den Studiengängen bis hin zur individuellen Ebene der Lehrenden. So konnte die hochschuldidaktische Begleitung der Studiengangentwicklung vorangetrieben und feste Formate hochschuldidaktischer Weiterbildungen etabliert werden. Damit wurde der durch die Bologna-Reform angestoßene Struktur- und Lehrkulturwandel an den Hochschulen in Bezug auf Hochschuldidaktik in konkreten Maßnahmen umgesetzt. Letztlich schaffte das Netzwerk die Grundlage für eine hochschuldidaktische Einheit an der Universität Leipzig, um Hochschuldidaktik aus einer Hand zu ermöglichen und klare Ansprechpersonen für Lehrende und mit Lehre befasste Mitarbeitende zu schaffen. Ein Kulturwandel hin zur Stärkung der Lehre wurde dadurch an der Universität Leipzig nachhaltig in Gang gesetzt.

\section{Zusammenfassung und Schlussfolgerungen}

Die zwei hier beispielhaft beschriebenen Netzwerke in StiL unterschieden sich in verschiedener Hinsicht, besonders bezüglich ihrer Gruppenzusammensetzung, ihres thematischen Profils und ihrer Zielstellungen. Gleichzeitig zeigten sie auf struktureller und organisatorischer Ebene viele Gemeinsamkeiten. Welche Schlussfolgerungen können daraus für die Bildung und Organisation zukünftiger Netzwerke gezogen werden?

\section{Initiierung und Entwicklung der Netzwerke}

Der Impuls zur Initiierung beider beispielhaft betrachteter Netzwerke ging zunächst von den Projektmitarbeitenden selbst aus. Zu Beginn handelte es sich in beiden Fällen um selbstorganisierte Netzwerke, die ihre Themen und Strukturen selbst setzten und entwickelten. Sie fußten auf dem gemeinsamen Interesse und Bedarf der jeweils Beteiligten, einen regelmäßigen Austausch in 
einem festen Netzwerk zu organisieren, um ähnliche Problemstellungen zu bearbeiten, Good-Practice-Beispiele auszutauschen, gegebenenfalls gemeinsame Projektvorhaben umzusetzen sowie Strategien durch Handlungsabstimmung zu entwickeln. So ging es anfangs vor allem darum, gemeinsame Schnittpunkte und Herausforderungen der Akteure zu identifizieren sowie die Ziele und die Ausrichtung des Netzwerks zu bestimmen.

Im Verlauf der Formierung und Konsolidierung der Netzwerke zeigte sich, dass die Gruppenzusammensetzung mit ihren unterschiedlichen strukturellen Anbindungen (zentral-dezentral), Aufgabenfeldern und Arbeitskontexten sowie individuellen und strategischen Ausrichtungen einen Einfluss auf die Netzwerkarbeit hatte. So gestaltete sich etwa die gemeinsame Zielsetzung sowie die thematische Fokussierung unterschiedlich. Im Hochschul- und Mediendidaktischen Netzwerk ging es beispielsweise einerseits um die Abstimmung und Synchronisierung bestehender sowie andererseits um die Entwicklung neuer hochschuldidaktischer Angebote zur Unterstützung der Lehrenden der Universität Leipzig. Hingegen sahen die Mitglieder des Mentoring-Netzwerks ihren Auftrag und ihre Aufgaben stärker in den Fakultäten verortet. Die Ziele bezogen sich hier mehr auf den Informations- und Erfahrungsaustausch.

In den beiden beispielhaft betrachteten Netzwerken war im Projektverlauf zu beobachten, dass sie unter anderem in Bezug auf die Intensität der Zusammenarbeit oder die inhaltliche Ausrichtung verschiedene Phasen durchliefen. So folgte der Formierungs- und Findungsphase im Mentoring-Netzwerk mit intensiver Zusammenarbeit und regelmäßigen Treffen eine Phase, die stärker den Austausch über die gesammelten Erfahrungen in konkreten Projekten, gegebenenfalls Transfer von Good Practice und die kollegiale Beratung in den Vordergrund rückte. Ähnliches war für das Hochschul- und Mediendidaktische Netzwerk zu beobachten. Hier wurde die Konsolidierung zudem durch die Hochschulleitung unterstützt und so in strategische Überlegungen der Hochschulentwicklung einbezogen. Die Zusammenarbeit der Netzwerkmitglieder wurde dadurch noch intensiviert. Solche Phasen engerer und weniger intensiver Zusammenarbeit waren auch in anderen Netzwerken im Rahmen von StiL (siehe Kapitel 2) zu beobachten, da sie sich stark an den Bedarfen der Teilnehmenden orientierten und daher immer wieder Anpassungen vorgenommen werden mussten.

\section{Intensive Vernetzung durch Projektarbeit}

Eine Gemeinsamkeit war weiterhin, dass die Netzwerkarbeit im Rahmen der Projektarbeit in StiL erfolgte, in dessen Rahmen Vernetzung einen hohen Stellenwert aufwies. So konnten Synergien geschaffen und Transfer von Good 
Practice ermöglicht werden. Entsprechend wurden Vernetzung und Austausch der Teilprojekte auch von der Projektleitung und vom Projektmanagement unterstützt und zeitliche und organisatorische Ressourcen für Austausch und Vernetzung eingeräumt und bereitgestellt (vgl. Kapitel 2). Das Mentoring-Netzwerk wurde auch seitens der Projektleitung und des Projektmanagements aktiv genutzt, unter anderem als Multiplikator für die Bewerbung von weiteren Maßnahmen in StiL, als Experimentierraum für neue Projektideen (vgl. Dimmer et alt. in diesem Band) oder für das Einbringen von Querschnittsthemen in die Fakultäten. Der Mehrwert bestand auch über das Netzwerk hinaus. Das Hochschul- und Mediendidaktische Netzwerk wurde dann besonders wirksam, als die Konsolidierung seitens der Hochschulleitung erfolgte, da das Netzwerk explizit auch Regelstrukturen, für die Austausch und Vernetzung nicht vorrangiger Auftrag waren, integrierte. Im Hochschul- und Mediendidaktischen Netzwerk waren vor allem die beiden QPL-Projekte LaborUniversität und LiT+ sehr aktiv an der Zusammenarbeit mit bestehenden Strukturen an der Universität interessiert. Ziel war die Schaffung der Hochschul- und Mediendidaktik aus einer Hand und die Vermeidung von Doppelstrukturen an der Universität Leipzig.

\section{Arbeitsweisen der Netzwerke}

In der Arbeitsweise blieben die Netzwerke selbstorganisiert und wiesen darin viele Gemeinsamkeiten auf. In beiden Netzwerken fanden regelmäßige Präsenztreffen statt. Für die Organisation, Moderation und Ergebnissicherung der Treffen wurden wechselnde Verantwortliche bestimmt. Zudem bildeten sich innerhalb des Netzwerks für punktuelle oder weiterführende Themen und Anliegen kleinere Arbeitsgruppen, die zwischen den Treffen - online oder vor Ort - ihren Austausch fortsetzten. Die Netzwerke nutzten zusätzlich Online-Plattformen, um die Dokumentation sowie weitere Absprachen zu organisieren. Durch die Online-Formate, konkrete Absprachen sowie die Bestimmung von Verantwortlichen konnte der räumlichen Verteilung der Netzwerke (Mentoring: Fakultäten der Universität Leipzig, Hochschul- und Mediendidaktisches Netzwerk: sachsen- und universitätsweit) gut begegnet werden.

Die Netzwerke trugen in einer gesamtuniversitären Perspektive zu Innovation sowie Diffusion neuer Lösungen bei. So konnten mit ihrer Hilfe gemeinsame Vorhaben wie Veranstaltungsformate, Workshops oder Mentoring-Programme und strategische Leitlinien entwickelt und umgesetzt werden. Diese dienten Studierenden, Lehrenden und Mitarbeitenden der Universität Leipzig gleichermaßen (Kulturwandel Verbesserung der Qualität der Lehre). Mit dem Hochschuldidaktischen Netzwerk und dem Mentoring-Netzwerk konnten neue Ser- 
vice-Einheiten sowohl zentral als auch dezentral an den Fakultäten geschaffen werden. Die Netzwerke waren zudem auch für andere Institutionen und Akteure sichtbar und die Expertise der Mitglieder wurde aktiv nachgefragt.

Aus den beiden vorgestellten Netzwerken lassen sich also Faktoren für eine gelingende Netzwerkarbeit schlussfolgern. Die Formulierung und Vereinbarung gemeinsamer Ziele zu Beginn tragen wesentlich zum Gelingen der Netzwerkarbeit bei. Im Projektverlauf können sich diese Ziele verändern und eine Anpassung notwendig machen. Die Konsolidierung seitens der Projekt- oder Hochschulleitung kann dabei zur Entwicklung und Stabilisierung der Netzwerke beitragen. Des Weiteren hängt die Umsetzung eines Netzwerks immer auch von der Initiative von Einzelpersonen oder -projekten ab, die das Netzwerk auch in Phasen weniger intensiver Zusammenarbeit am Leben halten („Gardening“, vgl. Lave / Wenger 1991). Netzwerkarbeit setzt eine gewisse Ergebnisoffenheit und Flexibilität der Mitglieder im Netzwerk voraus sowie ausreichend zeitliche und organisatorische Ressourcen. Dies sollte gegebenenfalls bereits im Rahmen der Planung von Projekten berücksichtigt werden. Wenn diese Aspekte bei der Netzwerkarbeit berücksichtigt werden, können sie sowohl für die Mitglieder als auch die Institutionen einen wichtigen Mehrwert leisten.

\section{Fazit}

Die zwei beschriebenen Netzwerke leisteten in ihrer Umsetzung einen wichtigen Beitrag zu einer erfolgreichen Entwicklung des Projekts StiL an der Universität Leipzig und wirkten auf vielfältige Weise in die Hochschule hinein. Die Netzwerke dienten nicht nur dazu, Wissen zu teilen und zu diskutieren, sondern konnten außerdem wichtige hochschulrelevante Arbeitsfelder wie Beratung, Qualitätsmanagement, Hochschuldidaktik und Projektmanagement weiter ausdifferenzieren, innovieren und professionalisieren.

Die Netzwerke wirkten durch ihre inhaltliche und strategische Ausrichtung auf unterschiedlichen Ebenen. Diese lassen sich sowohl aus der Perspektive der Netzwerkmitglieder als auch im Hinblick auf den gesamtuniversitären Kontext beschreiben.

Aus der Perspektive der Netzwerkmitglieder konnten die Netzwerke unter anderem dazu beitragen:

- Unsicherheiten, die zum Beispiel mit der Bearbeitung neuer Aufgabenbereiche verbunden waren, zu reduzieren,

- Handlungen abzustimmen,

- gute Praxisbeispiele bekannt zu machen, 
- Erfahrungsaustausch und Wissenstransfer zu ermöglichen und die Zusammenarbeit zu verbessern,

- Projekte und Vorhaben zu initiieren und umzusetzen sowie

- die individuelle professionelle Identität und Rollenfindung zu unterstützen.

Gleichzeitig konnten die Erfahrungen und Erkenntnisse aus den Netzwerken auf unterschiedlichen Ebenen in die jeweiligen Institutionen, Fakultäten oder Fachbereiche der Teilnehmenden hineinwirken.

Die Netzwerke wurden als Ansprechpartner für Querschnittsthemen erkannt und seitens weiterer Teilprojekte in StiL, aber auch seitens der Hochschulleitung und (teilweise initiiert durch das Projektmanagement) weiterer QPL-Projekte in Sachsen und deutschlandweit identifiziert und genutzt. Aus dem anfangs relativ losen Zusammenschluss interessierter Projektmitarbeitender entwickelten sich so feste Instanzen mit gemeinsamen Zielen, die sich im Projektverlauf zunehmend institutionalisierten. Projektarbeit konnte so Freiräume für Vernetzung schaffen und diese befördern. Durch die Unterstützung und Konsolidierung der Netzwerke durch die Projekt- und Hochschulleitung konnten sie ihre volle Wirksamkeit entfalten und so beispielsweise auch Mitarbeitende aus vorhandenen Regelstrukturen aktiv in die Netzwerke einbinden.

Eine Professionalisierung erfolgte im Projektverlauf innerhalb der Netzwerke auch im Hinblick auf ihre Arbeitsweise und ihre Organisation. So trugen die individuellen Kompetenzen der Mitarbeitenden, die sie entwickelten und in das Netzwerk einbrachten, sowie die regelmäßige Reflexion dieser Kompetenzen in den Projekten und Netzwerken wesentlich dazu bei.

Ein Weiterbestand der hier beispielhaft genannten Netzwerke, aber auch der weiteren Netzwerke, an denen StiL-Mitarbeitende beteiligt sind, ist dabei abhängig davon, ob Angebote, Aufgaben und Koordinationsarbeit, die bisher von QPL-Mitarbeitenden übernommen wurden, in die Zeit nach QPL verstetigt werden können.

\section{Literatur}

Bundesministerium für Bildung und Forschung (BMBF) (2020): Website des Ministeriums. Abgerufen am 07.05.2020, von: https://www.qualitaetspakt-lehre.de.

Deutsche Gesellschaft für Hochschuldidaktik (DGHD) (2020): Website der DGHD. Abgerufen am 07.05.2020, von: https://www.dghd.de.

Deutsche Gesellschaft für Hochschuldidaktik AG (DGHD AG) (2020): Qualitative Feedback- und Evaluationsmethoden. Abgerufen am 11.05.2020, von: https://www.dghd. de/community/arbeitsgruppen/g-qualitative-feedback-und-evaluationsmethoden/.

Kooperationsstelle Wissenschaft und Arbeitswelt (KOWA) (2020): Darstellung des Projekts Plan B. Abgerufen am 07.05.2020, von: https://kowa-leipzig.de/plan-b. 
Lave, J. / Wenger, E. (1991): Situated Learning: Legitimate Peripheral Participation, Cambridge: Cambridge University Press, 1991.

Lehren an der Universität Leipzig (2020): Website der Universität Leipzig. Abgerufen am 07.05.2020, von: https://www.uni-leipzig.de/universitaet/arbeiten-an-der-universitaetleipzig/lehren-an-der-universitaet/.

Moodle (2020): Zentrale Lernplattform der Universität Leipzig. Abgerufen am 07.05.2020, von: https://moodle2.uni-leipzig.de.

Qualitätspakt Lehre (QPL) (2020): Teilnehmende Hochschulen in Sachsen. Abgerufen am 20.04.2020, von: https://www.qualitaetspakt-lehre.de/de/geforderte-hochschulen-insachsen-1705.php.

Studieren in Leipzig (StiL) (2020a): Facharbeitskreis studentisches Peer-Learning. Abgerufen am 07.05.2020, von: https://www.stil.uni-leipzig.de/hochschuluebergreifendevernetzung/.

Studieren in Leipzig (StiL) (2020b): Tutoring-Leitlinie. Abgerufen am 20.04.2020, von: https://www.stil.uni-leipzig.de/wp-content/uploads/2018/11/Tutoring_Leitlinie_StiL2_ Juli_2016.pdf.

Universität Leipzig (2020): Tag der Lehre. Abgerufen am 07.05.2020, von: https://www.unileipzig.de/universitaet/arbeiten-an-der-universitaet-leipzig/lehren-an-der-universitaet/tagder-lehre/.

Dieser Text wurde von Mitarbeiterinnen dreier StiL-Teilprojekte verfasst:

\section{Mentoring}

StiL-Mentorinnen und -Mentoren sind an zwölf der 14 Fakultäten der Universität Leipzig angebunden. Ziel des Mentorings ist es, Studierende in allen Phasen des Studiums kompetent zu begleiten und zu beraten und so Studiengangwechsel und Studienabbrüche im Studienverlauf zu reduzieren. Die Mentorinnen und Mentoren haben fakultätsspezifische Arbeitsschwerpunkte. Darüber hinaus arbeiten sie in fakultätsübergreifenden Projekten zusammen.

\section{LaborUniversität}

Die Stil-LaborUniversität unterstützt Lehrende der Universität Leipzig bei der Entwicklung und Umsetzung innovativer Lehr-Lern-Projekte. Mit finanzieller Förderung und hochschuldidaktischer Begleitung wird den Lehrenden ermöglicht, ihre Ideen für eine lern- und kompetenzorientierte Lehre zu erproben. Weiterhin bietet die LaborUniversität individuelle Begleitung der Lehrkompetenzentwicklung für alle Lehrenden an der Universität Leipzig und unterstützt die Studiengangentwicklung. Sie arbeitet eng mit dem Verbundprojekt Lehrpraxis im Transfer plus (LiT+) zusammen.

\section{StiL-Projektmanagement}

Das Projektmanagement bietet Begleitung und Beratung bei der Projektdurchführung sowie der Konzeption von Maßnahmen und unterstützt die Kommunikation und Vernetzung. Die Sicherung der fördergemäßen Projektentwicklung durch Monitoring, Qualitätssicherung und Steuerung ist zentraler Auftrag. Das Projektmanagement vermittelt außerdem Projektergebnissen innerhalb von StiL und im Rahmen des Qualitätspakt Lehre. 